\title{
Default and Exogenous Collateral in Incomplete Markets with a Continuum of States
}

\author{
Jaime J. Orrillo C.* \\ Universidade Católica de Brasília \\ SGAN 916-Módulo B \\ 70790-000-Asa Norte, Brasília - DF \\ Brasil
}

\begin{abstract}
We consider an economy with a finite number of agents, a continuum of states and incomplete markets where individuals do not keep their promises contrary to the Arrow-Debreu model of general equilibrium with complete markets(GE) and the general equilibrium model with incomplete markets(GEI) where they do keep them by assumption. We show that in addition to usual assumptions on utilities and endowments the equilibrium for this economy must exists if sales of assets is backed by some nonzero collateral.
\end{abstract}

Keywords: Incomplete Markets; Asset Promises; Collateral; Durability

*This paper is part of my PhD thesis, written at IMPA in 1998. The author is deeply grateful to Aloisio Araujo by advice and encouragement and to Mario Pascoa by his useful suggestions and reading of the manuscript. Finally he also thanks the support from CNPQ Brazil.

e-mail: orrillo@pos.ucb.br; jjoc@impa.br; Fax: 55-61-340-5550 


\section{Introduction}

In the Arrow-Debreu model of general equilibrium with complete markets(GE) and likewise in the general equilibrium model with incomplete markets(GEI) agents keep all their promises by assumption. It is well known that in the later, without the lower bound on short sales, the equilibrium can fail to exist, see Hart (1975). However, Duffie and Shafer (1985) prove that Hart's example is held only on a null set, i.e. the equilibrium exists generically. Later Dubey et al. ( 1989,1990$)$ extend the GEI model to another where agents are not obligated to keep promises and they prove that the equilibrium always exists for this model whenever there exist a lower bound on short sales. As always this bound is necessary to guarantee the existence. On the other hand, when these models were extended for a continuum of states - see Mas-Collel and Monteiro (1996), Monteiro $(1995,1996)$ - in addition to the hypothesis of the lower bound short on sales it is required that ex-post endowments be nonnegative, this assumption is much stronger but indispensable, see MasColell and Zame (1996) and Monteiro (1996). Araújo, Monteiro and Páscoa (1997) eliminate this requirement by introducing default subject to utility penalties, in the set-up of Dubey et al. (1989).

The aim in this paper is to extend the pioneering study of collateral by Dubey-Geanakopolos-Zame(1995), where results on existence of equilibria are provided, for exogenously given collateral coefficients. We model the second period uncertainty by considering a probability space without atoms. Specifically, we consider $S \equiv[0,1]$ as the set of states of nature equipped by the sigma algebra of Lebesgue and of course the measure of Lebesgue. Moreover we make some simplifications which will not alter the arguments used in the proof on existence. For instance, we allow for each debtor ( seller of assets ) to hold the full collateral that secure the sale of asset, we also assume that the collateral, as well as any consumption good is durable. This 
durability is measured by a linear transformation depending on the state of nature that has been solved in the second period.

Penalties play nowadays a small deterrence role in the default world. Moreover in the presence of utility penalties, adverse selection problems may arise, since lenders do not know the default likelihood of borrowers, which depends on the comparison between marginal penalties and marginal utilities of income, in each state of nature. This asymmetric information context is richer but also harder to deal with than the anonymous model of collateralize promises without utility penalties.

The existence argument uses a finite-dimensional approximation and applies Fatou's lemma to a uniformly bounded sequence of equilibrium variables of the truncated economies. This mathematical approach of existence of equilibria with a continuum of states was already used in the non-default model of Hellwig ( 1996) and Monteiro (1996). This sequence of equilibria variables of truncated economic includes prices, allocations, portfolios and marginal utilities of income, as required to establish market clearing and optimality conditions in the limit economy. We prove that the only condition to guarantee the existence of the equilibrium, in addition to usual assumptions on utilities and endowments, is that the sales of assets should be backed by durable physical commodities (collateral). Essentially what happens is that this assumption helps to find a lower bound on short sales since the collateral is fixed. Collateral has the advantage that the lender need not bother with the reliability or even the identity of the borrower, but can concentrate entirely on the future value of the collateral. Collateral thus retains anonymity in market transactions. As we are assuming a model without penalties to defaulting, then every borrower will deliver the minimum of what he owes in every state and the value of the collateral he puts up to secure his promises.

The paper is organized as follows: in the next section we describe the 
model and state our result on existence of equilibria, in the section 3 we define the truncated economy which has equilibrium assumptions imposed by Dubey-Geanakopolos-Zame(1995). For each individual problem Slater's condition implies the existence of Lagrange multipliers, which will be shown to be bounded. Finally, in the last section I give the proof of the main theorem.

\section{The Model}

The economy has a finite number $H$ of agents and two periods. The uncertainty in the second period is modeled by a Lebesgue measure space $(S, \mathcal{S}, \lambda)$.

There are $L$ commodities and $J$ assets. In the first period each agent chooses a consumption vector $x \in \mathbb{R}_{+}^{L}$ and a portfolio of assets $z=\theta-\varphi$, where $\theta_{j} \geq 0$ is the number of units of asset $j$ that consumer buy and $\varphi_{j}$ is the number of units he sell. This sale should be backed by collection of goods (collateral) which will be put aside at the moment that assets are sold, even if the delivery is not scheduled for much later, and will be held by the borrower.

In the second period, consumption becomes given by a random variable $c: S \mapsto \mathbb{R}_{+}^{L}$ and the asset returns are given by a random variable $A: S \mapsto$ $\mathbb{R}_{+}^{J \times L}$. The collateral is even available in this period but depreciated. This depreciation is measured by mean of the random variable $Y: S \mapsto \mathbb{R}_{+}^{L \times L}$ or equivalently $\forall l \in L, \quad Y^{l}: S \mapsto \mathbb{R}_{+}^{L}$, where $Y_{s}^{l}$ represents the column the of matrix $Y_{s} \in \mathbb{R}_{+}^{L \times L}$ which will be assumed to be nonsingular diagonal.

Each agent is characterized by an initial endowment, $w^{h}=\left(e_{o}^{h}, e^{h}\right)$ with $e_{o}^{h} \in \mathbb{R}_{+}^{L}$ and $e^{h}: S \mapsto \mathbb{R}_{+}^{L}$ measurable and an utility function which is defined to be separable:

$$
U^{h}(x, c)=u_{0}(x)+\int_{S} u^{h}\left(s, c_{s}\right) \lambda(d s), \forall h \in H
$$


being $u_{0}^{h}(x)$ and $\int_{S} u^{h}\left(s, c_{s}\right) d s$ his pleasures derived from consumptions $x \in$ $\mathbb{R}_{+}^{L}$ in the first period, and $u^{h}(s,):. \mathbb{R}_{+}^{L} \mapsto \mathbb{R}$ in the second period respectively.

Let $\triangle_{n-1}$ be the $n$ - simplex in $\mathbb{R}^{n}$. Suppose that $(p, q, \pi)$ is the price system of the economy. More precisely, $p \in \triangle_{L-1}$ is the commodity-price vector in the first period; $q: S \mapsto \triangle_{L-1}$ is a random variable representing the prices of commodities at second period, and finally $\pi \in \triangle_{J-1}$ asset-price vector.

The borrower debt will be $q_{s} A_{s}^{j} \varphi_{j}^{h}$. Of this debt, he pays $D_{s}^{j}$ which is defined by $D_{s}^{j}:=\min \left\{q_{s} A_{s}^{j}, q_{s}\left[Y_{s} C_{j}\right]\right\}$ that is the total delivery of money on asset $j$ in state $s$. Similarly, each lender should expect to receive only the minimum between the claim and the value of the collateral. That is, $D_{s}^{j}$.

Thus, Our economy is defined by

$$
\mathcal{E}=\left(\left(U^{h}, w^{h}\right)_{h \in H},\left(A^{j}, C_{j}\right)_{j \in J},\left(Y^{l}\right)_{l \in L}\right)
$$

In this setting each agent $h \in H$ faces the following problem:

$$
\max _{(x, c, \theta, \varphi) \in B^{h}(p, q, \pi)} U^{h}\left(x+\sum_{j \in J} C_{j} \varphi_{j}, c\right),
$$

where $B^{h}(p, q, \pi)$ is the budget set defined by all the $(x, c, \theta, \varphi) \in \mathbb{R}_{+}^{L} \times$ $L^{\infty}\left(S, \mathbb{R}_{+}^{L}\right) \times \mathbb{R}_{+}^{J} \times \mathbb{R}_{+}^{J}$ satisfying the following:

$$
p x+\pi \theta+\sum_{j \in J} p C_{j} \varphi_{j} \leq p e_{o}^{h}+\pi \varphi
$$

and

$$
q_{s} c_{s}+\sum_{j \in J} D_{s}^{j} \varphi_{j}^{h} \leq q_{s} e_{s}^{h}+\sum_{j \in J} D_{s}^{j} \theta_{j}+q_{s} Y_{s} x+\sum_{j \in J} q_{s}\left[Y_{s} C_{j}\right] \varphi_{j} \text { a.e. }
$$

Next, we will define the concept of equilibrium for this economy and then we will statement our main result. 
Definition 1 An equilibrium for $\mathcal{E}$ is a vector of prices $(p, q, \pi)$ and an allocation $\left(x^{h}, c^{h}, \theta^{h}, \varphi^{h}\right)_{h \in H}$ such that the markets clear and the choice is optimal, that is:

$$
\begin{aligned}
& \text { 1. } \sum_{h \in H}\left(x^{h}+C \varphi^{h}\right)=\sum_{h \in H} e_{o}^{h} . \\
& \quad \sum_{h \in H} c_{s}^{h}=\sum_{h \in H}\left(e_{s}^{h}+\sum_{j \in H}\left[Y_{s} C_{j}\right] \varphi_{j}^{h}+Y_{s} x\right) \text { a.e. } \\
& \quad \sum_{h \in H} \theta^{h}=\sum_{h \in H} \varphi^{h} .
\end{aligned}
$$

Theorem 1 (Main Theorem) We suppose that for all asset $j$, there $i s C_{j} \neq 0$ and:

1. $u^{h}(s,):. \mathbb{R}_{+}^{L} \mapsto \mathbb{R}$ is concave, continuous and strictly monotone, for all $s \in S ; u_{0}^{h}: \mathbb{R}_{+}^{L} \mapsto \mathbb{R}$ is concave, continuous and strictly monotone.

2. $\exists \delta>0$ such that $e_{s}^{h} \geq(\delta, \ldots, \delta), \forall h \in H$ a.e. and $e_{0}^{h} \in \mathbb{R}_{++}^{L}$

3. $u^{h}(., v)$ is bounded random variable $\forall v \in \mathbb{R}^{L}$

4. $A^{j}, e^{h}, Y^{l}: S \mapsto \mathbb{R}_{+}^{L}$ are bounded r.v.; $\forall j \in J, \forall h \in H$, $\forall l \in L$.

Then $\mathcal{E}$ has equilibrium.

In the next section we define the truncated economy. The existence of equilibrium for this economy follows from the Geanakopolos - Dubey -Zame (1995). In this way we have a sequence of equilibria. Our goal is to show that its limit is an equilibrium for $\mathcal{E}$. The idea is to apply Fatou's Lemma $n$ - dimensional. For that it sufficient that the truncated economy equilibrium sequence is uniformly bounded. The only problem to establish the optimality condition in the limit economy is to control the Lagrange's multipliers associated to each individual problem. We bound them in lemmas 4 and 5 below. 


\section{The truncated economy}

$\mathcal{E}^{N 1}=\left(\left(U^{h N}, e_{o}^{h N}, e^{h N}\right)_{h \in H},\left(A_{j N}, C_{j}^{N}\right)_{j \in J},\left(Y^{N l}\right)_{l \in L}\right)$

\section{Utilities}

$$
\begin{aligned}
& U^{h N}: \mathbb{R}_{+}^{(N+1) L} \mapsto \mathbb{R} \text { is defined by } \\
& U^{h N}\left(x_{o}^{N},\left(x_{n}^{N}\right)_{n \in N}\right):=u_{0}^{h}\left(x_{o}^{N}\right)+\sum_{n=1}^{N} \int_{\frac{n-1}{N}}^{\frac{n}{N}} u^{h}\left(s, x_{n}^{N}\right) d s .
\end{aligned}
$$

\section{Endowments}

$$
\begin{aligned}
& \left(e_{o}^{h N},\left(e_{n}^{h N}\right)_{n \in N}\right) \text { where } \\
& e_{o}^{h N}:=e_{o}^{h}, \\
& e_{n}^{h N}:=N \int_{\frac{n-1}{N}}^{\frac{n}{N}} e_{s}^{h} d s .
\end{aligned}
$$

\section{Asset promises and collateral levels}

$$
\begin{aligned}
& A_{n}^{j N}:=N \int_{\frac{n-1}{N}}^{\frac{n}{N}} A_{s}^{j} d s \\
& C_{j}^{N}:=C_{j}
\end{aligned}
$$

\section{Durabilities of goods}

$Y_{n}^{l N}:=N \int_{\frac{n-1}{N}}^{\frac{n}{N}} Y_{s}^{l} d s$. Hence $Y^{l N} \in \mathbb{R}_{+}^{N L} ; Y^{N}: N \mapsto \mathbb{R}_{+}^{L \times L}$, i.e $Y_{n}^{N}$ is a matrix whose columns are $Y_{n}^{l N} \in \mathbb{R}_{+}^{L}, \forall l \in L$.

\section{Prices}

$$
\left(p_{o}^{N},\left(p_{n}^{N}\right)_{n \in N}, \pi^{N}\right) \in \triangle_{L-1}^{N+1} \times \triangle_{J-1}
$$

\section{Delivery}

$$
D_{n}^{j N}:=\min \left\{p_{n}^{N} A_{n}^{j N}, p_{n}^{N} Y_{n}^{N} C_{j}\right\} \in \mathbb{R}
$$

Lemma 1 The four hypothesis of the main theorem imply that $\mathcal{E}^{N}$ has equilibrium, that is, $\exists\left(\left(p_{n}^{N}\right)_{n=0,1, \ldots, N}, \pi^{N},\left(\left(x_{n}^{h N}\right)_{n=0,1, \ldots, N}, \theta^{h N}, \varphi^{h N}\right)_{h \in H}\right) \in \triangle_{L-1}^{N+1} \times$ $\triangle_{J-1} \times\left(\mathbb{R}_{+}^{(N+1) L} \times \mathbb{R}_{+}^{J} \times \mathbb{R}_{+}^{J}\right)^{H}$ such that is satisfied the following:

\footnotetext{
${ }^{1}$ The equilibrium for this economy is the same as before with $n$ for $s$
} 
1. $\sum_{h \in H}\left[x_{o}^{h N}+\sum_{j \in J} C_{j} \varphi_{j}^{h N}\right]=\sum_{h \in H} e_{o}^{h}$,

$\sum_{h \in H} x_{n}^{h N}=\sum_{h \in H}\left(e_{n}^{h N}+\sum_{j \in J}\left[Y_{n}^{N} C_{j}\right] \varphi_{j}^{h N}+Y_{n}^{N} x_{o}^{h N}\right), \forall n \in N$

$\sum_{h \in H} \theta^{h N}=\sum_{h \in H} \varphi^{h N}$.

2. $\left(x_{o}^{h N},\left(x_{n}^{h N}\right)_{n \in N}, \theta^{h N}, \varphi^{h N}\right)$ maximizes $U^{h N}$ on $B^{h}\left(p_{o}^{N},\left(p_{n}^{N}\right)_{n \in N}, \pi^{N}\right)$

Proof: The hypothesis 1, 2, 3 and 4 of the theorem 1 imply the hypothesis required for the existence of the equilibrium for $\mathcal{E}^{N} .^{2}$

Lemma 2 Lemma 1 implies there are Lagrange multipliers $\left(\mu_{n}^{h N}\right)_{n=0,1, \ldots, N} \in$ $\mathbb{R}_{+}^{N+1}$ such that:

1. $\mu_{o}^{h N}\left\{p_{o}^{N}\left(x_{o}^{h N}-e_{o}^{h N}\right)+\pi^{N}\left(\theta^{h N}-\varphi^{h N}\right)+p_{o}^{N} \sum_{j \in J} C_{j} \varphi_{j}^{h N}\right\}=0$

$\mu_{n}^{h N}\left\{p_{n}^{N}\left(x_{n}^{h N}-Y_{n}^{N} x_{o}^{h N}-e_{n}^{h N}\right)+\sum_{j \in J}\left(D_{n}^{j N}-p_{n}^{N}\left[Y_{n}^{N} C_{j}\right]\right) \varphi_{j}^{h N}-\sum_{j \in J} D_{n}^{j N} \theta_{j}^{h N}\right\}=$

$0, \forall n \in N$.

2. $u_{0}^{h}\left(x_{o}^{N}+C \varphi^{N}\right)-u_{0}^{h}\left(x_{o}^{h N}+C \varphi^{h N}\right)+\sum_{n=1}^{N} \int_{\frac{n-1}{N}}^{\frac{n}{N}}\left[u^{h}\left(s, x_{n}^{N}\right)-u^{h}\left(s, x_{n}^{h N}\right)\right] d s \leq$

$$
\begin{aligned}
& \mu_{o}^{h N}\left\{p_{o}^{N}\left(x_{o}^{N}-x_{o}^{h N}\right)+\pi^{N}\left[\left(\theta^{N}-\varphi^{N}\right)-\left(\theta^{h N}-\varphi^{h N}\right)\right]+\sum_{j \in J} p_{o}^{N} C_{j}\left(\varphi_{j}^{N}-\varphi_{j}^{h N}\right)\right\} \\
& +\sum_{n=1}^{N} \mu_{n}^{h N}\left\{p_{n}^{N}\left(x_{n}^{N}-x_{n}^{h N}\right)-p_{n}^{N} Y_{n}^{N}\left(x_{o}^{N}-x_{o}^{h N}\right)+\sum_{j \in J}\left(D_{n}^{j N}-p_{n}^{N}\left[Y_{n}^{N} C_{j}\right]\right)\left(\varphi_{j}^{N}-\right.\right. \\
& \left.\left.\varphi_{j}^{h N}\right)-\sum_{j \in J} D_{n}^{j N}\left(\theta_{j}^{N}-\theta_{j}^{h N}\right)\right\} \\
& \text { for all, } x_{o}^{N} \geq 0,\left(x_{n}^{N}\right)_{n \in N} \geq 0, \theta^{N} \geq 0, \varphi^{N} \geq 0
\end{aligned}
$$

Proof: 1 and 2 are direct consequences of the Kunh-Tucker theorem and Slater's condition (the null vector satisfy this condition).

Lemma 3 The following inequality is true:

$$
\int_{\frac{n-1}{N}}^{\frac{n}{N}}\left[u^{h}(s, x)-u^{h}\left(s, x_{n}^{h N}\right)\right] d s \leq \mu_{n}^{h N} p_{n}^{N}\left(x-x_{n}^{h N}\right), x \in \mathbb{R}_{+}^{L}, n=1, \ldots, N
$$

\footnotetext{
${ }^{2}$ See Dubey, Geanakoplos and Zame (1995)
} 


\section{Proof:}

Fix $m \in N$, then substituting $\theta^{N}=\theta^{h N}, \varphi^{N}=\varphi^{h N}, x_{o}^{N}=x_{o}^{h N}$ and $x_{n}^{N}=$ $x_{n}^{h N}, \forall n \neq m$. in the item 2 of the Lemma 2 we obtain

$$
\int_{\frac{m-1}{N}}^{\frac{m}{N}}\left[u^{h}\left(s, x_{m}^{N}\right)-u^{h}\left(s, x_{m}^{h N}\right)\right] d s \leq \mu_{m}^{h N} p_{m}^{N}\left(x_{m}^{N}-x_{m}^{h N}\right)
$$

Since $\mathrm{m}$ is arbitrary we obtain the desired inequality.

Lemma $4 \mu_{s}^{N h}:=N \mu_{n}^{h N}$, if $s \in\left[\frac{n-1}{N}, \frac{n}{N}\right), n \in N$, then $\left\{\mu^{h N}\right\}_{N}$ is a sequence of uniformly bounded functions.

\section{Proof:}

Applying $Y_{n}^{N}$ in the first equality of the item 1 of the lemma 1 and then by replacing $\sum_{h \in H} \sum_{j \in J} Y_{n}^{N} C_{j} \varphi_{j n}^{N}$ in the second equality of the item 1 of the same lemma we have $\sum_{h \in H} x_{n}^{h N}=\sum_{h \in H}\left(e_{n}^{h N}+Y_{n}^{N} e_{o}^{h}\right)$. Therefore $x_{n}^{h N} \leq$ $\sum_{h \in H}\left(e_{n}^{h N}+Y_{n}^{N} e_{o}^{h}\right)$ which is bounded, say by $\beta \in R_{+}^{L}$, since $e^{h}$ and $Y^{l}$ are bounded. Without loss of generality we can suppose that $u^{h}(s, 0)=0$, then by replacing $x=0$ in the inequality of the lemma 3 and by using the monoticity of $u^{h}(s,$.$) we have$

$$
\mu_{n}^{h N} p_{n}^{N} x_{n}^{h N} \leq \int_{\frac{n-1}{N}}^{\frac{n}{N}} u^{h}\left(s, x_{n}^{h N}\right) d s \leq \int_{\frac{n-1}{N}}^{\frac{n}{N}} u^{h}(s, \beta) d s
$$

From the second equality of the item 1 of the lemma 2 follows that

$\mu_{n}^{h N} p_{n}^{N} x_{n}^{h N}=\mu^{h N} p_{n}^{N} e_{n}^{h N}+\mu_{n}^{h N} p_{n}^{N} Y_{n}^{N} x_{o}^{h N}+\mu^{h N}\left\{D_{n}^{N} \theta^{h N}+\left(p_{n}^{N} Y_{n}^{N} C-D_{n}^{N}\right) \varphi^{h N}\right\}$

$\forall n=1,2, \ldots, N$. The right hand side is greater than $\mu^{h N} p_{n}^{N} e_{n}^{h N}$ since the other terms are positive. Hence $\mu^{h N} p_{n}^{N} e_{n}^{h N} \leq \mu_{n}^{h N} p_{n}^{N} x_{n}^{h N}$ and therefore (3) is transformed in

$$
\mu_{n}^{h N} p_{n}^{N} e_{n}^{h N} \leq \int_{\frac{n-1}{N}}^{\frac{n}{N}} u^{h}(s, \beta) d s
$$


Now, using the fact that $e_{s}^{h}>(\delta, \ldots, \delta) \in \mathbb{R}_{++}^{L}$ and $e_{n}^{h N}:=N \int_{\frac{n-1}{N}}^{\frac{n}{N}} e_{s}^{h} d s$ one obtains $e_{n}^{h N}>(\delta, \ldots, \delta) \in \mathbb{R}_{++}^{L}$. Using this fact and multiplying by $\frac{N}{\delta}$ in both sides of (3) we have

$$
N \mu_{n}^{h N} \leq \frac{N}{\delta} \int_{\frac{n-1}{N}}^{\frac{n}{N}} u^{h}\left(s, x_{n}^{h N}\right) d s .
$$

Therefore, for each $s \in\left[\frac{n-1}{N}, \frac{n}{N}\right)$ one has

$$
\mu_{s}^{h N} \leq \int_{\frac{n-1}{N}}^{\frac{n}{N}} u^{h}(s, \beta) d s \leq \frac{\sup _{s \in S} u^{h}(s, \beta)}{\delta} .
$$

Lemma 5 The sequence $\left\{\mu_{o}^{h N}\right\}_{N}$ is bounded.

\section{Proof:}

In the item 2 of the lemma 2 we replace $x_{n}^{N}=x_{n}^{h N} \forall n \in N, x_{o}^{N}=0, \theta^{N}=0$ and $\varphi^{N}=0$. So $u_{o}^{h}(0)-u_{o}^{h}\left(x_{o}^{h N}+C \varphi^{h N}\right) \leq-\mu_{o}^{h N}\left\{p_{o}^{N} x_{o}^{h N}+\pi^{N}\left(\theta^{h N}-\varphi^{h N}\right)\right.$ $\left.+\sum_{j \in J} p_{o}^{N} C_{j} \varphi_{j}^{h N}\right\}+\sum_{n=1}^{N} \mu_{n}^{h N}\left\{p_{n}^{N} Y_{n}^{N} x_{o}^{h N}-\sum_{j \in J}\left(D_{n}^{j N}-p_{n}^{N}\left[Y_{n}^{N} C_{j}\right]\right) \varphi_{j}^{h N}+\right.$ $\left.\sum_{j \in J} D_{n}^{j N} \theta_{j}^{h N}\right\}$

Now, using the item 1 of the lemma 2 we have

$$
u_{0}^{h}(0)-u_{0}^{h}\left(x_{o}^{h N}+C \varphi^{h N}\right) \leq-\mu^{h N} p_{o}^{N} e_{o}^{h N}+\sum_{n} \mu_{n}^{h N} p_{n}^{N}\left(x_{n}^{h N}-e_{n}^{h N}\right)
$$

After moving terms and using $e_{o}^{h N}=e_{o}^{h}$ and the fact that $x_{0 N}^{h}+C \varphi^{h N} \leq$ $\sum_{h}\left(x_{o}^{h N}+C \varphi^{h N}\right)=\sum_{h} e_{o}^{h}$ one has

$$
\begin{aligned}
\mu_{o}^{h N} p_{o}^{N} e_{o}^{h N} & \leq u_{0}^{h}\left(x_{o}^{h N}+C \varphi^{h N}\right)-u_{0}^{h}(0)+\sum_{n} \mu_{n}^{h N} p_{n}^{N}\left(x_{n}^{h N}-e_{n}^{h N}\right) \\
& \leq u_{o}^{h}\left(\sum_{h} e_{o}^{h}\right)-u_{0}^{h}(0)+\sum_{n} \mu_{n}^{h N} p_{n}^{N} x_{n}^{h N} \\
& \leq u_{o}^{h}\left(\sum_{h} e_{o}^{h}\right)-u_{0}^{h}(0)+\sum_{n \in N} \int_{\frac{n-1}{N}}^{\frac{n}{N}} u^{h}(s, \beta) d s
\end{aligned}
$$

Therefore

$$
\min _{l \in L} e_{o l}^{h} \mu_{o}^{h N} \leq u_{o}^{h}\left(\sum_{h} e_{o}^{h}\right)-u_{0}^{h}(0)+\sup _{s \in S} u^{h}(s, \beta)
$$


Hence

$$
\mu_{o}^{h N} \leq \frac{u_{o}^{h}\left(\sum_{h} e_{o}^{h}\right)-u_{0}^{h}(0)+\sup _{s \in S} u^{h}(s, \beta)}{\min _{l \in L} e_{o l}^{h}}
$$

as desired.

\section{The Proof of the Main Theorem}

Without loss of generality we can suppose that:

$\left(\pi^{N}, \mu_{o}^{h N}, \theta^{h N}, \varphi^{h N}, x_{o}^{h N}, p_{o}^{N}\right) \rightarrow\left(\pi, \mu_{0}^{h}, \theta^{h}, \varphi^{h}, x^{h}, p\right)$ when $N \rightarrow \infty$ since all these sequences are bounded. We also define the following sequences of simple functions:

$$
\begin{aligned}
c_{s}^{h N} & :=x_{n}^{h N} \text { if } s \in\left[\frac{n-1}{N}, \frac{n}{N}\right), q_{s}^{N}:=p_{n}^{N} \text { if } s \in\left[\frac{n-1}{N}, \frac{n}{N}\right), \\
e_{s}^{h N} & :=e_{n}^{h N} \text { if } s \in\left[\frac{n-1}{N}, \frac{n}{N}\right), A_{s}^{j N}:=A_{n}^{j N} \text { if } s \in\left[\frac{n-1}{N}, \frac{n}{N}\right) \text { and } \\
Y_{s}^{N} & :=Y_{n}^{N} \text { if } s \in\left[\frac{n-1}{N}, \frac{n}{N}\right) ; \text { for all } N \in \mathbb{N} \text { with } n \in N .
\end{aligned}
$$

The assumption 4 of the theorem 1 implies that sequences $q_{s}^{N}, e_{s}^{h N}, A_{s}^{j N}$ and $Y_{s}^{N}$ defined above are bounded. Therefore the expression $\sum_{h}\left(e_{n}^{h N}+Y_{n}^{N} e_{o}^{h}\right)$ is bounded as well. On the other hand, the two first equalities of the item 1 of the lemma 1 imply that $x_{n}^{h N} \leq \sum_{h}\left(e_{n}^{h N}+Y_{n}^{N} e_{o}^{h}\right)$. So the simple function $c^{h N}$ defined above is bounded. All this facts imply that the function $f_{N}: S \rightarrow \mathbb{R}^{G}$, ( $G$ is not specified) defined by

$$
f_{N}:=\left(\mu^{h N}, c^{h N}, q^{N}, \mu^{h N} q^{N} A_{N}^{j}, \mu^{h N} q^{N} Y^{N}, e^{h N}\right)_{h \in H, j \in J}
$$

is uniformly bounded, hence the hypothesis of the stronger version of Fatou's lemma are satisfied. Therefore $\exists f: S \rightarrow \mathbb{R}^{G}$ integrable such that:

$$
f_{s} \in \operatorname{cl}\left\{f_{N}(s)\right\} \text { for a.e s, }
$$

and

$$
\int f(s) d s=\lim _{N \rightarrow \infty} \int f_{N}(s) d s
$$


Coordinate by coordinate $f$ is given by $\left(\mu^{h}, c^{h}, q, \mu^{h} q A^{j}, \mu^{h} q Y, e^{h N}\right)_{h \in H, j \in J}$ Next, we will prove that $\left(p, q, \pi,\left(x^{h}, c^{h}, \theta^{h}, \varphi^{h}\right)_{h \in H}\right)$ is a equilibrium for $\mathcal{E}$. In fact:

\section{Claim 1}

Markets clear in $\mathcal{E}$

Proof:

We know that $\left(\left(p_{n}^{N}\right)_{n=0,1, \ldots, N}, \pi^{N},\left(\left(x_{n}^{h N}\right)_{n=0,1, \ldots, N}, \theta^{h N}, \varphi^{h N}\right)_{h \in H}\right)$ is a equilibrium for $\mathcal{E}^{N}$, therefore in particular holds the following:

$$
\begin{aligned}
& \sum_{h \in H} x_{o}^{h N}=\sum_{h \in H}\left(e_{o N}^{h}-\sum_{j \in J} C_{j} \varphi_{j}^{h N}\right) . \\
& \sum_{h \in H} x_{n}^{h N}=\sum_{h \in H}\left(e_{n}^{h N}+\sum_{j \in J}\left[Y_{n}^{N} C_{j}\right] \varphi_{j}^{h N}+Y_{n}^{N} x_{o}^{h N}\right), \forall n \in N \\
& \sum_{h \in H} \theta^{h N}=\sum_{h \in H} \varphi^{h N} .
\end{aligned}
$$

By taking limit when $N \rightarrow \infty$, we have that $\left(p, q, \pi,\left(x^{h}, c^{h}, \theta^{h}, \varphi^{h}\right)_{h \in H}\right)$ verify 1 and 2 of the definition of the equilibrium for $\mathcal{E}$. It remains to prove that the choice is optimal, more precisely we have

\section{Claim 2}

For each $h,\left(x^{h}, c^{h}, \theta^{h}, \varphi^{h}\right)$ maximizes $U^{h}$ on $B^{h}(p, q, \pi)$.

\section{Proof:}

Let $(x, c, \theta, \varphi) \in B^{h}(p, q, \pi)$ be any arbitrarily feasible allocation, i.e., $x \in$ $\mathbb{R}_{+}^{L}, c \in L^{\infty}\left(S, \mathbb{R}_{+}^{L}\right),(\theta, \varphi) \in \mathbb{R}_{+}^{2 J}$ such that (1) and (2) are satisfied, then we should verify that:

$$
U^{h}\left(x+\sum_{j \in J} C_{j} \varphi_{j}, c\right) \leq U^{h}\left(x^{h}+\sum_{j \in J} C_{j} \varphi_{j}^{h}, c^{h}\right)
$$

i.e.

$$
u_{0}^{h}\left(x+\sum_{j \in J} C_{j} \varphi_{j}\right)-u_{0}^{h}\left(x^{h}+\sum_{j \in J} C_{j} \varphi_{j}^{h}\right)+\int_{S} u^{h}\left(s, c_{s}\right) d s-\int_{S} u^{h}\left(s, c_{s}^{h}\right) d s \leq 0
$$

Let $\left\{x_{o}^{N}\right\},\left\{\theta^{N}\right\},\left\{\varphi^{N}\right\}$ be arbitrary sequences such that $x_{o}^{N} \rightarrow x, \theta^{N} \rightarrow$ $\theta, \varphi^{N} \rightarrow \varphi$. 
$c$ is an integrable function since it is bounded and $S$ has finite measure. By using this $c$, we define, for all $N \in \mathbb{N}, c_{s}^{N}:=N \int_{\frac{n-1}{N}}^{\frac{n}{N}} c_{s} d s$, if $s \in\left[\frac{n-1}{N}, \frac{n}{N}\right)$. Let us denote this number by $c_{n}^{N}$. Therefore Leguesgue's derivation theorem implies that $c^{N} \rightarrow c$, a.e. on $S$ what implies that $c^{N} \rightarrow c$ in measure. Therefore the hypothesis of the Lemma 6 ( see appendix) are satisfied. So $u\left(., c^{N}().\right) \rightarrow u(., c()$.$) in measure what implies that there exists a conver-$ gence subsequence, that is, $u\left(., c^{N_{j}}().\right) \rightarrow{ }^{3} u(., c()$.$) a.e. on S$

The fact $c \in L^{\infty}(S)$ implies that $c^{N}$ is bounded by $\|c\|_{\infty}$. As $u(s,$.$) is$ monotone and $u(., v)$ is bounded for all $v \in \mathbb{R}^{L}$ it follows that $\left\{u\left(s, c_{s}^{N}\right)\right\}$ is uniformly bounded, therefore uniformly integrable and as $u\left(., c^{N}().\right) \rightarrow$ $u(., c()$.$) a.e. on S$ one has

$$
\int u\left(s, c_{s}\right) d s=\lim _{N \rightarrow \infty} \int u\left(s, c_{s}^{N}\right) d s
$$

thus

$$
\int u\left(s, c_{s}\right) d s=\lim _{N \rightarrow \infty} \sum_{n=1}^{N} \int_{\frac{n-1}{N}}^{\frac{n}{N}} u\left(s, c_{s}^{N}\right) d s
$$

Now, by using the last equality, continuity of the $u_{o}^{h}$ and then applying the item 2 of the lemma 2 to the expression ( the second line below ) that is

\footnotetext{
${ }^{3}$ later we avoid the sub-indices for simplifying the notation
} 
inside the brackets, we have:

$$
\begin{aligned}
& u_{o}^{h}(x+C z)-u_{0}^{h}\left(x^{h}+C z^{h}\right)+\int_{S}\left[u^{h}\left(s, c_{s}\right)-u^{h}\left(s, c_{s}^{h}\right] d s\right. \\
& =\lim _{N \rightarrow \infty}\left\{u_{0}^{h}\left(x_{o}^{N}+\varphi^{N}\right)-u_{0}^{h}\left(x_{n}^{h N}+C z^{h N}\right)+\sum_{n=1}^{N} \int_{\frac{n-1}{N}}^{\frac{n}{N}}\left[u^{h}\left(s, c_{n}^{N}\right)-u^{h}\left(s, x_{n}^{h N}\right)\right] d s\right\} \\
& \leq \lim _{N \rightarrow \infty} \mu_{o}^{h N}\left\{p_{o}^{N}\left(x_{o}^{N}-x_{o}^{h N}\right)+\pi^{N}\left[\left(\theta^{N}-\theta^{h N}\right)-\left(\varphi^{N}-\varphi^{h N}\right)\right]\right. \\
& \left.+\sum_{j \in J} p_{o}^{N} C_{j}\left(\varphi_{j}^{N}-\varphi_{j}^{h N}\right)\right\}+\lim _{N \rightarrow \infty} \sum_{n=1}^{N} \mu_{n}^{h N}\left\{p_{n}^{N}\left(x_{n}^{N}-x_{n}^{h N}\right)-p_{n}^{N} Y_{n}^{N}\left(x_{o}^{N}-x_{o}^{h N}\right)\right. \\
& \left.+\sum_{j \in J}\left(D_{n}^{j N}-p_{n}^{N}\left[Y_{n}^{N} C_{j}\right]\right)\left(\varphi_{j}^{N}-\varphi_{j}^{h N}\right)-\sum_{j \in J} D_{n}^{j N}\left(\theta_{j}^{N}-\theta_{j}^{h N}\right)\right\}
\end{aligned}
$$

The first limit on the right side hand is zero for both $(x, \theta, \varphi)$ and $\left(x^{h}, \theta^{h}, \varphi^{h}\right)$ satisfy the first budget constraint, is that, they satisfy (1). In the second limit the only that we can use is the feasibility of $\left(x_{n}^{h N}, \theta_{j}^{h N}, \varphi_{j}^{h N}\right)_{n \in N}$. More precisely satisfies the truncated second budget constraint. Therefore

$$
\begin{aligned}
U^{h}(x+C z, c)-U^{h}\left(x^{h}+C z^{h}, c^{h}\right) & \leq \lim _{N \rightarrow \infty} \sum_{n=1}^{N} \mu_{n}^{h N}\left\{p_{n}^{N}\left(c_{n}^{N}-e_{n}^{h N}-Y_{n}^{N} x_{o}^{N}\right)\right. \\
& \left.+\sum_{j \in J}\left(D_{n}^{j N}-p_{n}^{N}\left[Y_{n}^{N} C_{j}\right]\right) \varphi_{j}^{N}-\sum_{j \in J} D_{n}^{j N} \theta_{j}^{N}\right\}
\end{aligned}
$$

After multiplying $N$ and dividing $\frac{1}{N}$ simultaneously in the right hand side of the last inequality we obtain

$$
\begin{aligned}
& \lim _{N \rightarrow \infty} \sum_{n=1}^{N} N \mu_{n}^{h N}\left\{p_{n}^{N}\left(c_{n}^{N}-e_{n}^{h N}-Y_{n}^{N} x_{o}^{N}\right)+\sum_{j \in J}\left(D_{n}^{j N}-p_{n}^{N}\left[Y_{n}^{N} C_{j}\right]\right) \varphi_{j}^{N}\right. \\
& \left.-\sum_{j \in J} D_{n}^{j N} \theta_{j}^{N}\right\} \frac{1}{N}
\end{aligned}
$$

Now, By definition of functions $\mu^{h N}, q^{N}, C^{N}, e^{h N}$ and $Y^{N}$ one has that 
the previous expression is equal to

$$
\begin{aligned}
& \lim _{N \rightarrow \infty} \sum_{n=1}^{N} \int_{\frac{n-1}{N}}^{\frac{n}{N}} \mu_{s}^{h N}\left\{q_{s}^{N}\left(c_{s}^{N}-e_{s}^{h N}-Y_{s}^{N} x_{o}^{N}\right)+\sum_{j \in J}\left(D_{s}^{j N}-q_{s}^{N}\left[Y_{s}^{N} C_{j}\right]\right) \varphi_{j}^{N}\right. \\
& \left.-\sum_{j \in J} D_{s}^{j N} \theta_{j}^{N}\right\} \\
& =\lim _{N \rightarrow \infty} \int_{S} \mu_{s}^{h N}\left\{q_{s}^{N}\left(c_{s}^{N}-e_{s}^{h N}-Y_{s}^{N} x_{o}^{N}\right)+\sum_{j \in J}\left(D_{s}^{j N}-q_{s}^{N}\left[Y_{s}^{N} C_{j}\right]\right) \varphi_{j}^{N}\right. \\
& \left.-\sum_{j \in J} D_{s}^{j N} \theta_{j}^{N}\right\}
\end{aligned}
$$

Finally, using (8) and after the feasibility of $(x, c, \theta, \varphi)$ one has that the right hand side of the last equality is

$$
\int_{S} \mu_{s}^{h}\left\{q_{s}\left(c_{s}-e_{s}^{h}-Y_{s} x\right)+\sum_{j \in J}\left(D_{s}^{j}-q_{s}\left[Y_{s} C_{j}\right]\right) \varphi_{j}-\sum_{j \in J} D_{s}^{j} \theta_{j}\right\} \leq 0 .
$$

Hence

$$
U^{h}\left(x+\sum_{j \in J} C_{j} \varphi_{j}, c\right) \leq U^{h}\left(x^{h}+\sum_{j \in J}\left(C_{j} \varphi_{j}^{h}, c^{h}\right)\right.
$$

as desired.

\section{Appendix}

In the proof of the main theorem we used a result of measure convergence involving a function $u(s, x)$ with $s \in S$ and $x \in \mathbb{R}_{+}^{L}$ having the following conditions ${ }^{4}$ :

1. $u(s,):. \mathbb{R}_{+}^{L} \rightarrow \mathbb{R}$ is continuous for almost all $s \in S$ and

2. $u(., x): S \rightarrow \mathbb{R}$ is measurable for all $x \in \mathbb{R}_{+}^{L}$.

\footnotetext{
${ }^{4}$ This condition are called Caratheodory conditions.
} 
Lemma 6 Let the operator $\mathbf{f}$ defined on the set of all functions $c: S \rightarrow \mathbb{R}_{+}^{L}$ by the equation $\mathbf{f} c(s)=u(s, c(s))$ where $u(.,$.$) satisfies the Caratheodory con-$ ditions. Then the operator $\mathbf{f}$ transforms every sequence of functions which converges in measure $\left\{c^{N}(s)\right\}$ into a sequence of functions which also converges in measure.

Proof: The lemma follows from the lemma 2.1 in Krasnosel'skii (1964, p. 20)

\section{References}

[1] Araújo A., P. Monteiro and M. Páscoa, 1997, "Incomplete Markets, Continuum of states and Default", Economic Theory

[2] Araújo A., M. R. Páscoa and J. Orrillo, 1998, "Existence of Equilibrium with Default and Endogenous Collateral", Working Paper IMPA.

[3] Bewley, Jr. F., 1972, "Existence of equilibria with infinitely many commodities", Journal of Economic Theory 4, 514-540.

[4] Dubey, Pr., J. Geanakoplos (1989), Liquidity and bankruptcy with incomplete markets: Pure exchange; Cowels Foundation discussion paper 900 .

[5] Dubey, P. and Geanakoplos, 1990. "Default and Efficiency in a General Equilibrium Model with Incomplete Markets" Cowles Foundation Discussion Paper, No. 773R.

[6] Duffie, D., 1996, "Incomplete security markets with infinitely many states: An introduction", Journal of Mathematical Economics 26, 1-8. 
[7] Duffie, D., 1990, "The nature of incomplete security markets", Graduate School of Business, Stanford University.

[8] Duffie D. and Shafer W., 1985. "Equilibrium in incomplete markets I: A basic model of generic existence", Journal of Mathematical Economics $14,285-300$.

[9] Duffie D. and Shafer W., 1986a. "Equilibrium in incomplete markets II: Generic Existence in Stochastic Economies", Journal of Mathematical Economics 15, 199-216.

[10] Geanakoplos, J., W. Zame, and R. Dubey, 1995. "Default, Collateral, and Derivatives", Yale University, mimeo.

[11] Geanakoplos, John, 1996. "Promises Promises", Cowles Foundation, Yale University.

[12] Hart, O., 1975. "On the optimality of equilibrium when the market structure is incomplete", Journal of Economic Theory 11, 418-443.

[13] Hellwig, M., 1996. "Rational expectations equilibria in sequence economies with symetric information: two period case", Journal of Mathematical Economics 26 No. 1, 9-50.

[14] Hildenbrand, W., 1974, "Core and equilibria of a large economy", Princeton University Press, Princeton.

[15] Krasnosel'skii, 1964, Topological methods in the theory of nonlinear integral equations (McMillan, New York )

[16] Mas-Colell, A. and P.K. Monteiro, 1996. "Self-fulfilling equilibria: An existence theorem for a general state space", Journal of Mathematical Economics 26, 51-62. 
[17] Mas-Colell, A. and W. Zame, 1996, "The existence of security markets equilibria with a non-atomic state space, journal of Mathematical Economics $26,63-84$

[18] Monteiro, P.K., 1995, "Uma concretização de um contra-examplo de Mas-Colell e Zame", Revista de Econometria 15, 65-75.

[19] Monteiro, P.K., 1996, "A new proof of existence of equilibrium in incomplete markets economies", Journal of Mathematics Economics 26, 85-101.

[20] Orrillo, J.J, 1998, "Exogenous Collateral", Working Paper, IMPA.

[21] Radner, R., 1972, "Existence of equilibrium of plans, prices and prices expectations in a sequence of markets", Econometrica 40, 289-303.

[22] Werner, J., 1985, "Equilibrium in economies with incomplete financial markets", Journal of Economic Theory 36, 110-119.

[23] Zame, W., 1993, "Efficiency and the role of default when security markets are Incomplete", American Economic Review 83, 1142-1164. 